\title{
ON THE FREQUENCY OF DRAWDOWNS FOR BROWNIAN MOTION PROCESSES
}

\author{
DAVID LANDRIAULT *** AND \\ BIN LI, ${ }^{* * * *}$ University of Waterloo \\ HONGZHONG ZHANG, ${ }^{* * * *}$ Columbia University
}

\begin{abstract}
Drawdowns measuring the decline in value from the historical running maxima over a given period of time are considered as extremal events from the standpoint of risk management. To date, research on the topic has mainly focused on the side of severity by studying the first drawdown over a certain prespecified size. In this paper we extend the discussion by investigating the frequency of drawdowns and some of their inherent characteristics. We consider two types of drawdown time sequences depending on whether a historical running maximum is reset or not. For each type we study the frequency rate of drawdowns, the Laplace transform of the $n$th drawdown time, the distribution of the running maximum, and the value process at the $n$th drawdown time, as well as some other quantities of interest. Interesting relationships between these two drawdown time sequences are also established. Finally, insurance policies protecting against the risk of frequent drawdowns are also proposed and priced.
\end{abstract}

Keywords: Drawdown; frequency; Brownian motion

2010 Mathematics Subject Classification: Primary 60G40

Secondary 60J65; 91B24

\section{Introduction}

We consider a drifted Brownian motion $X=\left\{X_{t}, t \geq 0\right\}$, defined on a filtered probability space $\left(\Omega,\left\{\mathcal{F}_{t}, t \geq 0\right\}, \mathbb{P}\right)$, with dynamics

$$
X_{t}=x_{0}+\mu t+\sigma W_{t},
$$

where $x_{0} \in \mathbb{R}$ is the initial value, $\mu \in \mathbb{R}, \sigma>0$, and $\left\{W_{t}, t \geq 0\right\}$ is a standard Brownian motion. The time of the first drawdown over size $a>0$ is denoted by

$$
\tau_{a}:=\inf \left\{t>0: M_{t}-X_{t} \geq a\right\},
$$

where $M=\left\{M_{t}, t \geq 0\right\}$ with $M_{t}:=\sup _{s \in[0, t]} X_{t}$ is the running maximum process of $X$. Throughout, we follow the convention that inf $\varnothing=\infty$ and $\sup \varnothing=0$.

Drawdown is one of the most frequently quoted path-dependent risk indicators for mutual funds and commodity trading advisers (see, e.g. Burghardt et al. [4]). From a risk management

\footnotetext{
Received 8 October 2013; revision received 1 March 2014.

* Postal address: Department of Statistics and Actuarial Science, University of Waterloo, Waterloo, ON, N2L 3G1, Canada.

** Email address: dlandria@uwaterloo.ca

*** Email address: bin.li@uwaterloo.ca

**** Postal address: Department of Statistics, Columbia University, New York, NY 10027, USA. Email address: hzhang@stat.columbia.edu
} 
standpoint, large drawdowns should be considered as extreme events of which both the severity and the frequency need to be investigated. Considerable attention has been paid to the severity aspect of the problem by prespecifying a threshold, namely $a>0$, of the size of drawdowns, and subsequently studying various properties associated to the first drawdown time $\tau_{a}$. In this paper we extend the discussion by investigating the frequency of drawdowns. To this end, we derive the joint distribution of the $n$th drawdown time, the running maximum, and the value process at the drawdown time for a drifted Brownian motion. Using the general theory on renewal processes, we proceed to characterize the behavior of the frequency of drawdown episodes in a long time-horizon. Finally, we introduce some insurance policies which protect against the risk associated with frequent drawdowns. These policies are similar to the sequential barrier options in the over-the-counter (OTC) market (see, e.g. Pfeffer [16]). Through Carr's randomization of maturities, we provide closed-form pricing formulas by making use of the main theoretical results of the paper.

\subsection{Literature review}

The first drawdown time $\tau_{a}$ is the first passage time of the drawdown process $\left\{M_{t}-X_{t}, t \geq 0\right\}$ to level $a$ or above. It has been extensively studied in the literature of applied probability. The joint Laplace transform of $\tau_{a}$ and $M_{\tau_{a}}$ was first derived by Taylor [20] for a drifted Brownian motion. Lehoczky [13] extended the results to a general time-homogeneous diffusion by a perturbation approximation approach. An infinite series expansion of the distribution of $\tau_{a}$ was derived by Douady et al. [8] for a standard Brownian motion and the results were generalized to a drifted Brownian motion by Magdon et al. [14]. The dual of drawdown, known as drawup, measures the increase in value from the historical running minimum over a given period of time. The probability that a drawdown precedes a drawup was subsequently studied by Hadjiliadis and Večeř [10] and Pospisil et al. [18] under the drifted Brownian motion and the general timehomogeneous diffusion process, respectively. Mijatovic and Pistorius [15] derived the joint Laplace transform of $\tau_{a}$ and the last passage time at level $M_{\tau_{a}}$ prior to $\tau_{a}$, associated with the joint distribution of the running maximum, the running minimum, and the overshoot at $\tau_{a}$ for a spectrally negative Lévy process. The probability that a drawdown precedes a drawup in a finite time-horizon was studied under drifted Brownian motions and simple random walks in [24]. More recently, Zhang [23] and Zhang and Hadjiliadis [25] studied Laplace transforms of the drawdown time, the so-called speed of market crash, and various occupation times at the first exit and the drawdown time for a general time-homogeneous diffusion process.

In quantitative risk management, drawdowns and its descendants have become an increasingly popular and relevant class of path-dependent risk indicators. A portfolio optimization problem with constraints on drawdowns was explicitly solved by Grossman and Zhou [9] in a Black-Scholes framework. Hamelink and Hoesli [11] used the relative drawdown as a performance measure in the optimization of real estate portfolios. Chekhlov et al. [6] proposed a new family of risk measures called conditional drawdown and studied parameter selection techniques and portfolio optimization under constraints on conditional drawdown. Some novel financial derivatives were introduced by Večeř [21] to the hedge maximum drawdown risk. Pospisil and Večeř [17] invented a class of 'Greeks' (where the sensitivity of options in a portfolio are denoted by Greek letters) to study the sensitivity of investment portfolios to running maxima and drawdowns. Later, Carr et al. [5] introduced a class of European-style digital drawdown insurances and proposed semistatic hedging strategies using barrier options and vanilla options. The swap type insurances and cancelable insurances against drawdowns were studied in Zhang et al. [26]. 


\subsection{Definitions}

While sustaining downside risk can be appropriately characterized using the drawdown process and the first drawdown time, economic turmoil and volatile market fluctuations are better described by quantities containing more pathwise information, such as the frequency of drawdowns. The existing knowledge about the first drawdown time $\tau_{a}$ provides only limited and implicit information about the frequency of drawdowns. For the purpose of tackling the problem of frequency directly and systematically, we define below two types of drawdown time sequences depending on whether the last running maximum needs to be recovered or not.

The first sequence $\left\{\tilde{\tau}_{a}^{n}, n \in \mathbb{N}\right\}$ is called the drawdown times with recovery, defined recursively as

$$
\tilde{\tau}_{a}^{n}:=\inf \left\{t>\tilde{\tau}_{a}^{n-1}: M_{t}-X_{t} \geq a, M_{t}>M_{\tilde{\tau}_{a}^{n-1}}\right\},
$$

where $\tilde{\tau}_{a}^{0}=0$. Note that, after each $\tilde{\tau}_{a}^{n-1}$, the corresponding running maximum $M_{\tilde{\tau}_{a}^{n-1}}$ must be recovered before the next drawdown time $\tilde{\tau}_{a}^{n}$. In other words, the running maximum is reset and updated only when the previous one is revisited. Since the sample paths of $X$ are almost surely (a.s.) continuous, it follows that $M_{\tilde{\tau}_{a}^{n}}-X_{\tilde{\tau}_{a}^{n}}=a$ a.s. if $\tilde{\tau}_{a}^{n}<\infty$.

The second sequence $\left\{\tau_{a}^{n}, n \in \mathbb{N}\right\}$ is called the drawdown times without recovery, defined recursively as

$$
\tau_{a}^{n}:=\inf \left\{t>\tau_{a}^{n-1}: M_{\left[\tau_{a}^{n-1}, t\right]}-X_{t} \geq a\right\}
$$

where $\tau_{a}^{0}:=0$ and $M_{[s, t]}:=\sup _{s \leq u \leq t} X_{u}$. From definition (1.3), it is implicitly assumed that the running maximum $M_{\tau_{a}^{n}}$ is 'reset' to $X_{\tau_{a}^{n}}$ at the drawdown time $\tau_{a}^{n}$. In fact, $\tau_{a}^{n}$ is the so-called iterated stopping times associated with $\tau_{a}$ defined as

$$
\tau_{a}^{n}= \begin{cases}\tau_{a}^{n-1}+\tau_{a} \circ \theta_{\tau_{a}^{n-1}} & \text { when } \tau_{a}^{n-1} \text { and } \tau_{a} \circ \theta_{\tau_{a}^{n-1}} \text { are finite } \\ \infty & \text { otherwise }\end{cases}
$$

where $\theta$ is the Markov shift operator such that $X_{t} \circ \theta_{s}=X_{s+t}$ for $s, t \geq 0$.

Note that both $\tau_{a}^{n}$ and $\tilde{\tau}_{a}^{n}$ are independent of the initial value $x_{0}$ for not only the drifted Brownian motion $X$, but also a general Lévy process. In view of definitions (1.2) and (1.3), it is clear that the following inclusive relation of the two types of drawdown time holds:

$$
\left\{\tilde{\tau}_{a}^{n}, n \in \mathbb{N}\right\} \subset\left\{\tau_{a}^{n}, n \in \mathbb{N}\right\} .
$$

In other words, for each $n \in \mathbb{N}$, there exists a unique positive integer $m \geq n$ such that $\tilde{\tau}_{a}^{n}=\tau_{a}^{m}$ (if $\tilde{\tau}_{a}^{n}<\infty$ ).

Our motivation for introducing the two drawdown time sequences is as follows. The drawdown times with recovery $\left\{\tilde{\tau}_{a}^{n}, n \in \mathbb{N}\right\}$ are easy to identify from the sample paths of $X$ by searching the running maxima. Moreover, they are consistent with definition (1.1) of the first drawdown $\tau_{a}$ in the sense that a drawdown can be considered as incomplete if the running maximum has not been revisited. However, there are also some crucial drawbacks of $\left\{\tilde{\tau}_{a}^{n}, n \in \mathbb{N}\right\}$ which motivate us to introduce the drawdown times without recovery $\left\{\tau_{a}^{n}, n \in \mathbb{N}\right\}$. First, the downside risk during recovering periods is neglected. One or more larger drawdowns may occur in a recovering period. Secondly, the threshold $a$ needs to be adjusted in order to gain a more integrated understanding about the severity of drawdowns. In other words, the selection of $a$ becomes tricky. Thirdly, the requirement of recovery is too strong. In the real world, a historical high water mark may never be recovered again, as in the case of a financial bubble (see, e.g. [12]). 
The rest of the paper is organized as follows. In Section 2 some preliminaries on exit times and the first drawdown time $\tau_{a}$ of the drifted Brownian motion $X$ are presented. In Section 3 the frequency rate of drawdowns, and the Laplace transform of $\tilde{\tau}_{a}^{n}$ associated with the distribution of $M_{\tilde{\tau}_{a}^{n}}$ and/or $X_{\tilde{\tau}_{a}^{n}}$ are derived. Section 4 is parallel to Section 3 but concerns the drawdown times without recovery $\left\{\tau_{a}^{n}, n \in \mathbb{N}\right\}$. Interesting connections between the two drawdown time sequences are established. In Section 5 some insurance contracts are introduced to insure against the risk of frequent drawdowns.

\section{Preliminaries}

Henceforth, for ease of notation, we write $\mathbb{E}_{x_{0}}[\cdot]=\mathbb{E}\left[\cdot \mid X_{0}=x_{0}\right]$ for the conditional expectation, $\mathbb{P}_{x_{0}}\{\cdot\}$ for the corresponding probability, and $\mathbb{E}_{x_{0}}[\cdot ; U]=\mathbb{E}_{x_{0}}\left[\cdot \mathbf{1}_{U}\right]$ with $\mathbf{1}_{U}$ denoting the indicator function of a set $U \subset \Omega$. In particular, when $x_{0}=0$, we drop the subscript $x_{0}$ from the conditional expectation and probability.

For $x \in \mathbb{R}$, let $T_{x}^{+}=\inf \left\{t \geq 0: X_{t}>x\right\}$ and $T_{x}^{-}=\inf \left\{t \geq 0: X_{t}<x\right\}$ be the first passage times of $X$ to levels in $(x, \infty)$ and $(-\infty, x)$, respectively. For $a<x<b$ and $\lambda>0$, it is known that

$$
\mathbb{E}_{x}\left[\mathrm{e}^{-\lambda T_{a}^{-}}\right]=\mathrm{e}^{\beta_{\lambda}^{-}(x-a)} \text { and } \mathbb{E}_{x}\left[\mathrm{e}^{-\lambda T_{b}^{+}}\right]=\mathrm{e}^{\beta_{\lambda}^{+}(x-b)},
$$

where $\beta_{\lambda}^{ \pm}=\left(-\mu \pm \sqrt{\mu^{2}+2 \lambda \sigma^{2}}\right) / \sigma^{2}$ (see, e.g. Equation 2.0.1, of Borodin and Salminen [3, p. 295]). By letting $\lambda \rightarrow 0+$ in (2.1), it follows that

$$
\mathbb{P}_{x}\left\{T_{b}^{+}<\infty\right\}=\mathrm{e}^{\left((-\mu+|\mu|) / \sigma^{2}\right)(x-b)} \quad \text { and } \quad \mathbb{P}_{x}\left\{T_{a}^{-}<\infty\right\}=\mathrm{e}^{\left((-\mu-|\mu|) / \sigma^{2}\right)(x-a)} .
$$

From Taylor [20] or Equation (17) of Lehoczky [13], we obtain the following joint Laplace transform of the first drawdown time $\tau_{a}$ and its running maximum $M_{\tau_{a}}$.

Lemma 2.1. For $\lambda, s>0$, it holds that

$$
\mathbb{E}\left[\mathrm{e}^{-\lambda \tau_{a}-s M_{\tau_{a}}}\right]=\frac{c_{\lambda}}{b_{\lambda}+s}
$$

where $b_{\lambda}=\left(\beta_{\lambda}^{+} \mathrm{e}^{-\beta_{\lambda}^{-} a}-\beta_{\lambda}^{-} \mathrm{e}^{-\beta_{\lambda}^{+} a}\right) /\left(\mathrm{e}^{-\beta_{\lambda}^{-} a}-\mathrm{e}^{-\beta_{\lambda}^{+} a}\right)$ and $c_{\lambda}=\left(\beta_{\lambda}^{+}-\beta_{\lambda}^{-}\right) /\left(\mathrm{e}^{-\beta_{\lambda}^{-} a}-\mathrm{e}^{-\beta_{\lambda}^{+} a}\right)$.

A Laplace inversion of (2.3) with respect to $s$ results in

$$
\mathbb{E}\left[\mathrm{e}^{-\lambda \tau_{a}} ; M_{\tau_{a}}>x\right]=\frac{c_{\lambda}}{b_{\lambda}} \mathrm{e}^{-b_{\lambda} x}
$$

for $x>0$. Furthermore, letting $x \rightarrow 0+$ in (2.4), we immediately have

$$
\mathbb{E}\left[\mathrm{e}^{-\lambda \tau_{a}}\right]=c_{\lambda} / b_{\lambda}
$$

A numerical evaluation of the distribution function of $\tau_{a}$ (and more generally $\tau_{a}^{n}$ and $\tilde{\tau}_{a}^{n}$ ) by an inverse Laplace transform method will be given at the end of Section 4. Other forms of infinite series expansion of the distribution of $\tau_{a}$ were derived by Douady et al. [8] and Magdon et al. [14] for a standard Brownian motion and a drifted Brownian motion, respectively. By taking the derivative with respect to $\lambda$ in (2.5) and letting $\lambda \rightarrow 0+$, it follows that

$$
\mathbb{E}\left[\tau_{a}\right]=\frac{\sigma^{2} \mathrm{e}^{2 \mu a / \sigma^{2}}-\sigma^{2}-2 \mu a}{2 \mu^{2}} .
$$


It is straightforward to check that

$$
\lim _{\lambda \rightarrow 0+} b_{\lambda}=\lim _{\lambda \rightarrow 0+} c_{\lambda}=\frac{\gamma}{\mathrm{e}^{\gamma a}-1},
$$

where $\gamma=2 \mu / \sigma^{2}$. In the literature on risk theory, the constant $\gamma$ is known as the adjustment coefficient. In particular, when $\mu=0$, the quantity $\gamma /\left(\mathrm{e}^{\gamma a}-1\right)$ is understood as $\lim _{\gamma \rightarrow 0} \gamma /\left(\mathrm{e}^{\gamma a}-1\right)=1 / a$. It follows from $(2.5)$ and (2.6) that

$$
\mathbb{P}\left\{\tau_{a}<\infty\right\}=\lim _{\lambda \rightarrow 0+} \mathbb{E}\left[\mathrm{e}^{-\lambda \tau_{a}}\right]=1 .
$$

Furthermore, it follows that

$$
\mathbb{P}\left\{M_{\tau_{a}} \geq x\right\}=\mathbb{P}\left\{M_{\tau_{a}} \geq x, \tau_{a}<\infty\right\}=\lim _{\lambda \rightarrow 0+} \mathbb{E}\left[\mathrm{e}^{-\lambda \tau_{a}} ; M_{\tau_{a}} \geq x\right]=\mathrm{e}^{-\gamma x /\left(\mathrm{e}^{\gamma a}-1\right)},
$$

which implies that the running maximum at the first drawdown time $M_{\tau_{a}}$ follows an exponential distribution with mean $\left(\mathrm{e}^{\gamma a}-1\right) / \gamma$ (see, e.g. [13]).

\section{The drawdown times with recovery}

We begin our analysis with the drawdown times with recovery $\left\{\tilde{\tau}_{a}^{n}, n \in \mathbb{N}\right\}$, given that their structure leads to a simpler analysis than their counterpart drawdown times without recovery.

We first consider the asymptotic behavior of the frequency rate of drawdowns with recovery. Let $\tilde{N}_{t}^{a}=\sum_{n=1}^{\infty} \mathbf{1}_{\left\{\tilde{\tau}_{a}^{n} \leq t\right\}}$ be the number of drawdowns with recovery observed by time $t \geq 0$, and define $\tilde{N}_{t}^{a} / t$ to be the frequency rate of drawdowns. It is clear that $\left\{\tilde{N}_{t}^{a}, t \geq 0\right\}$ is a delayed renewal process where the first drawdown time is distributed as $\tau_{a}$, while the subsequent inter-drawdown times are independent and identically distributed as $T_{X_{\tau_{a}}+a}^{+} \circ \tau_{a}$. From Theorem 6.1 .1 of Rolski et al. [19], it follows that, with probability 1,

$$
\lim _{t \rightarrow \infty} \frac{\tilde{N}_{t}^{a}}{t}= \begin{cases}\frac{1}{\mathbb{E}\left[\tau_{a}\right]+\mathbb{E}\left[T_{a}^{+}\right]}=\frac{2 \mu^{2}}{\sigma^{2}\left(\mathrm{e}^{2 \mu a / \sigma^{2}}-1\right)} & \text { if } \mu>0, \\ 0 & \text { if } \mu \leq 0 .\end{cases}
$$

Moreover, one could easily obtain some central limit theorems for $\tilde{N}_{t}^{a}$ by Theorem 6.1.2 of [19].

Next, we study the joint Laplace transform of $\tilde{\tau}_{a}^{n}$ and $M_{\tilde{\tau}_{a}^{n}}$. Note that $X_{\tilde{\tau}_{a}^{n}}=M_{\tilde{\tau}_{a}^{n}}-a$ a.s. whenever $\tilde{\tau}_{a}^{n}<\infty$, and, thus, the following theorem is sufficient to characterize the triplet $\left(\tilde{\tau}_{a}^{n}, M_{\tilde{\tau}_{a}^{n}}, X_{\tilde{\tau}_{a}^{n}}\right)$.

Theorem 3.1. For $n \in \mathbb{N}$ and $\lambda, x \geq 0$, it follows that

$$
\mathbb{E}\left[\mathrm{e}^{-\lambda \tilde{\tau}_{a}^{n}} ; M_{\tilde{\tau}_{a}^{n}}>x\right]=\left(\frac{c_{\lambda}}{b_{\lambda}}\right)^{n} \mathrm{e}^{-(n-1) \beta_{\lambda}^{+} a} \sum_{m=0}^{n-1} \frac{\left(b_{\lambda} x\right)^{m}}{m !} \mathrm{e}^{-b_{\lambda} x} .
$$

Proof. To prove this result, we first condition on the first drawdown time $\tau_{a}$ and subsequently on the time for the process $X$ to recover its running maximum. Using the strong Markov property of $X$ and (2.3), it is clear that

$$
\begin{aligned}
\mathbb{E}\left[\mathrm{e}^{-\lambda \tilde{\tau}_{a}^{n}-s M_{\tilde{\tau}_{a}^{n}}}\right] & =\mathbb{E}\left[\mathrm{e}^{-\lambda \tilde{\tau}_{a}^{n}-s M_{\tilde{\tau}_{a}^{n}}^{n}} ; \tilde{\tau}_{a}^{n}<\infty\right] \\
& =\mathbb{E}\left[\mathrm{e}^{-\lambda \tau_{a}-s M_{\tau_{a}}}\right] \mathbb{E}\left[\mathrm{e}^{-T_{a}^{+}}\right] \mathbb{E}\left[\mathrm{e}^{-\lambda \tilde{\tau}_{a}^{n-1}-s M_{\tilde{\tau}_{a}^{n-1}}}\right] \\
& =\frac{c_{\lambda}}{b_{\lambda}+s} \mathrm{e}^{-\beta_{\lambda}^{+} a} \mathbb{E}\left[\mathrm{e}^{-\lambda \tilde{\tau}_{a}^{n-1}-s M_{\tilde{\tau}_{a}^{n-1}}}\right]
\end{aligned}
$$




$$
\begin{aligned}
& =\left(\frac{c_{\lambda}}{b_{\lambda}+s}\right)^{n-1} \mathrm{e}^{-(n-1) \beta_{\lambda}^{+} a} \mathbb{E}\left[\mathrm{e}^{-\lambda \tau_{a}-s M_{\tau_{a}}}\right] \\
& =\left(\frac{c_{\lambda}}{b_{\lambda}+s}\right)^{n} \mathrm{e}^{-(n-1) \beta_{\lambda}^{+} a} .
\end{aligned}
$$

Given that $\left(b_{\lambda} /\left(b_{\lambda}+s\right)\right)^{n}$ is the Laplace transform of an Erlang random variable (RV) with mean $n / b_{\lambda}$ and variance $n /\left(b_{\lambda}\right)^{2}$, a tail inversion of (3.2) with respect to $s$ yields (3.1).

In particular, letting $x \rightarrow 0+$, it follows that

$$
\mathbb{E}\left[\mathrm{e}^{-\lambda \tilde{\tau}_{a}^{n}}\right]=\left(\frac{c_{\lambda}}{b_{\lambda}}\right)^{n} \mathrm{e}^{-(n-1) \beta_{\lambda}^{+} a}
$$

for $n \in \mathbb{N}$. Furthermore, letting $\lambda \rightarrow 0+$ in (3.3), together with (2.6) and $\lim _{\lambda \rightarrow 0+} \beta_{\lambda}^{+}=$ $(-\mu+|\mu|) / \sigma^{2}$, it follows that

$$
\mathbb{P}\left\{\tilde{\tau}_{a}^{n}<\infty\right\}= \begin{cases}1 & \text { if } \mu \geq 0 \\ \mathrm{e}^{(n-1) \gamma a} & \text { if } \mu<0 .\end{cases}
$$

In other words, a historical running maximum may never be recovered if the drift $\mu<0$.

Corollary 3.1. For $n \in \mathbb{N}$ and $x>0$, it holds that

$$
\mathbb{P}\left\{M_{\tilde{\tau}_{a}^{n}}>x, \tilde{\tau}_{a}^{n}<\infty\right\}= \begin{cases}\mathrm{e}^{-\gamma x /\left(\mathrm{e}^{\gamma a}-1\right)} \sum_{m=0}^{n-1} \frac{1}{m !}\left(\frac{\gamma x}{\mathrm{e}^{\gamma a}-1}\right)^{m} & \text { if } \mu \geq 0, \\ \mathrm{e}^{(n-1) \gamma a} \mathrm{e}^{-\gamma x /\left(\mathrm{e}^{\gamma a}-1\right)} \sum_{m=0}^{n-1} \frac{1}{m !}\left(\frac{\gamma x}{\mathrm{e}^{\gamma a}-1}\right)^{m} & \text { if } \mu<0 .\end{cases}
$$

Proof. Substituting (3.3) into (3.1) yields

$$
\mathbb{E}\left[\mathrm{e}^{-\lambda \tilde{\tau}_{a}^{n}} ; M_{\tilde{\tau}_{a}^{n}}>x\right]=\mathbb{E}\left[\mathrm{e}^{-\lambda \tilde{\tau}_{a}^{n}}\right] \sum_{m=0}^{n-1} \frac{\left(b_{\lambda} x\right)^{m}}{m !} \mathrm{e}^{-b_{\lambda} x} .
$$

Taking the limit when $\lambda \rightarrow 0+$ in (3.6), and then using (2.6), we arrive at

$$
\mathbb{P}\left\{M_{\tilde{\tau}_{a}^{n}}>x, \tilde{\tau}_{a}^{n}<\infty\right\}=\mathbb{P}\left\{\tilde{\tau}_{a}^{n}<\infty\right\} \sum_{m=0}^{n-1} \frac{\left(\gamma x /\left(\mathrm{e}^{\gamma a}-1\right)\right)^{m}}{m !} \mathrm{e}^{-\gamma x /\left(\mathrm{e}^{\gamma a}-1\right)} .
$$

Substituting (3.4) into (3.7) results in (3.5).

Note that (3.7) indicates

$$
\mathbb{P}\left\{M_{\tilde{\tau}_{a}^{n}}>x \mid \tilde{\tau}_{a}^{n}<\infty\right\}=\sum_{m=0}^{n-1} \frac{1}{m !}\left(\frac{\gamma x}{\mathrm{e}^{\gamma a}-1}\right)^{m} \mathrm{e}^{-\gamma x /\left(\mathrm{e}^{\gamma a}-1\right)}
$$

for all $\mu \in \mathbb{R}$. This result can be interpreted probabilistically. Indeed, when $\tilde{\tau}_{a}^{n}<\infty$, $M_{\tilde{\tau}_{a}^{m}}-M_{\tilde{\tau}_{a}^{m-1}}$ follows an exponential distribution with mean $\left(\mathrm{e}^{\gamma a}-1\right) / \gamma$ for $m=1,2, \ldots, n$. From the strong Markov property, the RVs $M_{\tilde{\tau}_{a}^{m}}-M_{\tilde{\tau}_{a}^{m-1}}$ for all $m=1,2, \ldots, n$ are all independent, and, thus, $M_{\tilde{\tau}_{a}^{n}}=\sum_{m=1}^{n}\left(M_{\tilde{\tau}_{a}^{m}}-M_{\tilde{\tau}_{a}^{m-1}}\right)$ is an Erlang RV with survival function (3.8). 
In particular, when $n \rightarrow \infty$, it is easy to check that $\lim _{n \rightarrow \infty} \mathbb{P}\left\{M_{\tilde{\tau}_{a}^{n}}>x\right\}=\mathbb{P}\left\{T_{x}^{+}<\infty\right\}$ which agrees with (2.2). For completeness, we conclude this section with a result that is immediate from (3.1) and the fact that $M_{\tilde{\tau}_{a}^{n}}-X_{\tilde{\tau}_{a}^{n}}=a$ a.s. whenever $\tilde{\tau}_{a}^{n}<\infty$.

Corollary 3.2. For $n \in \mathbb{N}$ and $x \geq-a$, it holds that

$$
\mathbb{E}\left[\mathrm{e}^{-\lambda \tilde{\tau}_{a}^{n}} ; X_{\tilde{\tau}_{a}^{n}}>x\right]=\left(\frac{c_{\lambda}}{b_{\lambda}}\right)^{n} \mathrm{e}^{-(n-1) \beta_{\lambda}^{+} a} \sum_{m=0}^{n-1} \frac{\left(b_{\lambda}(x+a)\right)^{m}}{m !} \mathrm{e}^{-b_{\lambda}(x+a)} .
$$

\section{Drawdown times without recovery}

In this section we turn our attention to the drawdown times without recovery. These are more challenging to analyze than their counterparts with recovery.

Let $N_{t}^{a}=\sum_{n=1}^{\infty} \mathbf{1}_{\left\{\tau_{a}^{n} \leq t\right\}}$ be the number of drawdowns without recovery by time $t \geq 0$. Clearly, $\left\{N_{t}^{a}, t \geq 0\right\}$ is a renewal process with independent inter-drawdown times, all distributed as $\tau_{a}$. By Theorem 6.1.1 of [19], it follows that, with probability 1,

$$
\lim _{t \rightarrow \infty} \frac{N_{t}^{a}}{t}=\frac{1}{\mathbb{E}\left[\tau_{a}\right]}=\frac{2 \mu^{2}}{\sigma^{2} \mathrm{e}^{2 \mu a / \sigma^{2}}-\sigma^{2}-2 \mu a},
$$

which is consistent with our intuition based on (1.4). Here again, we can also obtain some central limit theorems for $N_{t}^{a}$ by an application of Theorem 6.1.2 of [19].

Next, we characterize the joint distribution of $\left(\tau_{a}^{n}, X_{\tau_{a}^{n}}\right)$ by deriving an explicit expression for $\mathbb{E}\left[\mathrm{e}^{-\lambda \tau_{a}^{n}} ; X_{\tau_{a}^{n}}>x\right]$.

Theorem 4.1. For $n \in \mathbb{N}$ and $\lambda, x>0$, the joint distribution of $\left(\tau_{a}^{n}, X_{\tau_{a}^{n}}\right)$ satisfies

$$
\mathbb{E}\left[\mathrm{e}^{-\lambda \tau_{a}^{n}} ; X_{\tau_{a}^{n}}>x\right]=\left(\frac{c_{\lambda}}{b_{\lambda}}\right)^{n} \mathrm{e}^{-b_{\lambda}(x+n a)} \sum_{m=0}^{n-1} \frac{\left(b_{\lambda}(x+n a)\right)^{m}}{m !} .
$$

Proof. Given that $X_{\tau_{a}^{n}}+n a$ is a positive RV (and $X_{\tau_{a}^{n}}$ is not), we prove (4.1) by first deriving an expression for the joint Laplace transform of $\left(\tau_{a}^{n}, X_{\tau_{a}^{n}}+n a\right)$. By conditioning on the first drawdown time and its associated value process, and by making use of the strong Markov property and (2.3), it is clear that, for all $s \geq 0$,

$$
\begin{aligned}
\mathbb{E}\left[\mathrm{e}^{-\lambda \tau_{a}^{n}-s\left(X_{\tau_{a}^{n}}+n a\right)}\right] & =\mathbb{E}\left[\mathrm{e}^{-\lambda \tau_{a}-s\left(X_{\tau_{a}}+a\right)}\right] \mathbb{E}\left[\mathrm{e}^{-\lambda \tau_{a}^{n-1}-s\left(X_{\tau_{a}^{n-1}}+(n-1) a\right)}\right] \\
& =\mathbb{E}\left[\mathrm{e}^{-\lambda \tau_{a}-s M_{\tau_{a}}}\right] \mathbb{E}\left[\mathrm{e}^{-\lambda \tau_{a}^{n-1}-s\left(X_{\tau_{a}^{n-1}}+(n-1) a\right)}\right] \\
& =\frac{c_{\lambda}}{b_{\lambda}+s} \mathbb{E}\left[\mathrm{e}^{-\lambda \tau_{a}^{n-1}-s\left(X_{\left.\tau_{a}^{n-1}+(n-1) a\right)}\right.}\right] \\
& =\left(\frac{c_{\lambda}}{b_{\lambda}+s}\right)^{n} .
\end{aligned}
$$

The Laplace transform inversion of (4.2) with respect to $s$ results in

$$
\mathbb{E}\left[\mathrm{e}^{-\lambda \tau_{a}^{n}} ;\left(X_{\tau_{a}^{n}}+n a\right) \in \mathrm{d} y\right]=\left(c_{\lambda}\right)^{n} \frac{y^{n-1} \mathrm{e}^{-b_{\lambda} y}}{(n-1) !} \mathrm{d} y
$$

for $y \geq 0$. Integrating (4.3) over $y$ from $x+n a$ to $\infty$ yields (4.1). 
Letting $s \rightarrow 0+$ in (4.2), it follows that

$$
\mathbb{E}\left[\mathrm{e}^{-\lambda \tau_{a}^{n}}\right]=\left(\frac{c_{\lambda}}{b_{\lambda}}\right)^{n}=\left(\mathbb{E}\left[\mathrm{e}^{-\lambda \tau_{a}}\right]\right)^{n} .
$$

Note that (4.4) and (2.6) imply that

$$
\mathbb{P}\left\{\tau_{a}^{n}<\infty\right\}=1
$$

It is worth pointing out that the relation $\mathbb{E}\left[\mathrm{e}^{-\lambda \tau_{a}^{n}}\right]=\left(\mathbb{E}\left[\mathrm{e}^{-\lambda \tau_{a}}\right]\right)^{n}$ holds more generally for an $X$ that is a general Lévy process or a renewal risk process (also known as the Sparre Andersen risk model [2]), given that the inter-drawdown times $\tau_{a}^{1}$, and $\left\{\tau_{a}^{n}-\tau_{a}^{n-1}\right\}_{n \geq 2}$ form a sequence of independent and identically distributed (i.i.d.) RVs.

Similarly, letting $\lambda \rightarrow 0+$ in (4.1), it follows that

$$
\mathbb{P}\left\{X_{\tau_{a}^{n}} \geq x\right\}=\mathrm{e}^{-\gamma(x+n a) /\left(\mathrm{e}^{\gamma a}-1\right)} \sum_{m=0}^{n-1} \frac{\left(\gamma(x+n a) /\left(\mathrm{e}^{\gamma a}-1\right)\right)^{m}}{m !}
$$

for $n \in \mathbb{N}$ and $x \geq-n a$. As expected, (4.5) is the survival function of an Erlang RV with mean $n\left(\mathrm{e}^{\gamma a}-1\right) / \gamma$ and variance $n\left(\left(\mathrm{e}^{\gamma a}-1\right) / \gamma\right)^{2}$, later translated by $-n a$ units.

Our objective is now to include $M_{\tau_{a}^{n}}$ in the analysis of the $n$th drawdown time. A result particularly useful to this end is provided in Lemma 4.1, which considers a specific constrained Laplace transform of the first passage time to level $x$.

Lemma 4.1. For $n \in \mathbb{N}$ and $x>0$, the constrained Laplace transform of $T_{x}^{+}$together with this first passage time occurring before $\tau_{a}^{n}$ is given by

$$
\mathbb{E}\left[\mathrm{e}^{-\lambda T_{x}^{+}} ; T_{x}^{+}<\tau_{a}^{n}\right]=\mathrm{e}^{-b_{\lambda} x} \sum_{j=0}^{n-1}\left(c_{\lambda} \mathrm{e}^{-b_{\lambda} a}\right)^{j} \frac{x(x+j a)^{j-1}}{j !} .
$$

Proof. We prove this result by induction on $n$. For $n=1$, it follows that

$$
\begin{aligned}
\mathbb{E}\left[\mathrm{e}^{-\lambda T_{x}^{+}} ; T_{x}^{+}<\tau_{a}^{1}\right] & =\mathbb{E}\left[\mathrm{e}^{-\lambda T_{x}^{+}}\right]-\mathbb{E}\left[\mathrm{e}^{-\lambda T_{x}^{+}} ; T_{x}^{+}>\tau_{a}^{1}\right] \\
& =\mathrm{e}^{-\beta_{\lambda}^{+} x}-\int_{0}^{x} \mathbb{E}\left[\mathrm{e}^{-\lambda \tau_{a}^{1}} ; M_{\tau_{a}^{1}} \in \mathrm{d} y\right] \mathbb{E}_{y-a}\left[\mathrm{e}^{-\lambda T_{x}^{+}}\right] \\
& =\mathrm{e}^{-\beta_{\lambda}^{+} x}-\int_{0}^{x} c_{\lambda} \mathrm{e}^{-b_{\lambda} y} \mathrm{e}^{-\beta_{\lambda}^{+}(x-y+a)} \mathrm{d} y \\
& =\mathrm{e}^{-\beta_{\lambda}^{+} x}-c_{\lambda} \mathrm{e}^{-\beta_{\lambda}^{+} a} \frac{\mathrm{e}^{-\beta_{\lambda}^{+} x}-\mathrm{e}^{-b_{\lambda} x}}{b_{\lambda}-\beta_{\lambda}^{+}}
\end{aligned}
$$

where we use (2.4) in the third equality.

On the other hand, using the fact that $c_{\lambda} \mathrm{e}^{-\beta_{\lambda}^{+} a}=b_{\lambda}-\beta_{\lambda}^{+}$, it follows that

$$
\mathbb{E}\left[\mathrm{e}^{-\lambda T_{x}^{+}} ; T_{x}^{+}<\tau_{a}^{1}\right]=\mathrm{e}^{-b_{\lambda} x} .
$$


We now assume that (4.6) holds for $n=1,2, \ldots, k-1$ and that (4.6) also holds for $n=k$. Indeed, by the total probability equation,

$$
\begin{aligned}
\mathbb{E}\left[\mathrm{e}^{-\lambda T_{x}^{+}} ; T_{x}^{+}<\tau_{a}^{k}\right] & =\mathbb{E}\left[\mathrm{e}^{-\lambda T_{x}^{+}} ; T_{x}^{+}<\tau_{a}^{1}\right]+\mathbb{E}\left[\mathrm{e}^{-\lambda T_{x}^{+}} ; \tau_{a}^{1}<T_{x}^{+}<\tau_{a}^{k}\right] \\
& =\mathrm{e}^{-b_{\lambda} x}+\int_{0}^{x} \mathbb{E}\left[\mathrm{e}^{-\lambda \tau_{a}} ; M_{\tau_{a}} \in \mathrm{d} y\right] \mathbb{E}_{y-a}\left[\mathrm{e}^{-\lambda T_{x}^{+}} ; T_{x}^{+}<\tau_{a}^{k-1}\right] \mathrm{d} y \\
& =\mathrm{e}^{-b_{\lambda} x}+\int_{0}^{x} c_{\lambda} \mathrm{e}^{-b_{\lambda} y} \mathbb{E}\left[\mathrm{e}^{-\lambda T_{x-y+a}^{+}} ; T_{x-y+a}^{+}<\tau_{a}^{k-1}\right] \mathrm{d} y .
\end{aligned}
$$

Substituting (4.6) at $n=k-1$ into (4.7) yields

$$
\begin{aligned}
& \mathbb{E}\left[\mathrm{e}^{-\lambda T_{x}^{+}} ; T_{x}^{+}<\tau_{a}^{k}\right] \\
& \quad=\mathrm{e}^{-b_{\lambda} x}+c_{\lambda} \mathrm{e}^{-b_{\lambda}(x+a)} \sum_{j=0}^{k-2} \int_{0}^{x}\left(c_{\lambda} \mathrm{e}^{-b_{\lambda} a}\right)^{j} \frac{(x-y+a)(x-y+(j+1) a)^{j-1}}{j !} \mathrm{d} y \\
& =\mathrm{e}^{-b_{\lambda} x}+c_{\lambda} \mathrm{e}^{-b_{\lambda}(x+a)}\left(x+\sum_{j=1}^{k-2}\left(c_{\lambda} \mathrm{e}^{-b_{\lambda} a}\right)^{j} \int_{0}^{x}\left(\frac{(y+(j+1) a)^{j}}{j !}\right.\right. \\
& \left.=\mathrm{e}^{-b_{\lambda} x}\left(1+c_{\lambda} \mathrm{e}^{-b_{\lambda} a} x+\sum_{j=2}^{k-1}\left(c_{\lambda} \mathrm{e}^{-b_{\lambda} a}\right)^{j} \frac{(y+(j+1) a)^{j-1}}{(j-1) !}\right) \mathrm{d} y\right) \\
& =\mathrm{e}^{-b_{\lambda} x} \sum_{j=0}^{k-1}\left(c_{\lambda} \mathrm{e}^{-b_{\lambda} a}\right)^{j} \frac{x(x+j a)^{j-1}}{j !} .
\end{aligned}
$$

This completes the proof.

In the next theorem we provide a distributional characterization of the $n$th drawdown time $\tau_{a}^{n}$ with respect to both $M_{\tau_{a}^{n}}$ and $X_{\tau_{a}^{n}}$.

Theorem 4.2. For $n \in \mathbb{N}$ and $x>0$, it follows that

$$
\begin{aligned}
& \mathbb{E}\left[\mathrm{e}^{-\lambda \tau_{a}^{n}} ; M_{\tau_{a}^{n}}>x, X_{\tau_{a}^{n}} \in \mathrm{d} y\right] \\
& =\left(c_{\lambda}\right)^{n} \mathrm{e}^{-b_{\lambda}(y+n a)} \\
& \quad \times \sum_{m=0}^{n-1} \frac{x(x+m a)^{m-1}(y-x+(n-m) a)^{n-1-m} \mathbf{1}_{\{y-x+(n-m) a \geq 0\}}}{m !(n-m-1) !} \mathrm{d} y .
\end{aligned}
$$

Proof. By conditioning on the drawdown episode during which the drifted Brownian motion process $X$ reaches level $x$ for the first time and subsequently using the strong Markov property, 
it follows that

$$
\begin{aligned}
& \mathbb{E}\left[\mathrm{e}^{-\lambda \tau_{a}^{n}} ; M_{\tau_{a}^{n}}>x, X_{\tau_{a}^{n}} \in \mathrm{d} y\right] \\
& \quad=\sum_{m=0}^{n-1} \mathbb{E}\left[\mathrm{e}^{-\lambda \tau_{a}^{n}} ; M_{\tau_{a}^{n}}>x, X_{\tau_{a}^{n}} \in \mathrm{d} y, \tau_{a}^{m}<T_{x}^{+}<\tau_{a}^{m+1}\right] \\
& \quad=\sum_{m=0}^{n-1} \mathbb{E}\left[\mathrm{e}^{-\lambda T_{x}^{+}} ; \tau_{a}^{m}<T_{x}^{+}<\tau_{a}^{m+1}\right] \mathbb{E}_{x}\left[\mathrm{e}^{-\lambda \tau_{a}^{n-m}} ; X_{\tau_{a}^{n-m}} \in \mathrm{d} y\right] .
\end{aligned}
$$

From Lemma 4.1, we know that

$$
\begin{aligned}
\mathbb{E}\left[\mathrm{e}^{-\lambda T_{x}^{+}} ; \tau_{a}^{m}<T_{x}^{+}<\tau_{a}^{m+1}\right] & =\mathbb{E}\left[\mathrm{e}^{-\lambda T_{x}^{+}} ; \tau_{a}^{m}<T_{x}^{+}\right]-\mathbb{E}\left[\mathrm{e}^{-\lambda T_{x}^{+}} ; \tau_{a}^{m+1}<T_{x}^{+}\right] \\
& =\left(c_{\lambda}\right)^{m} \frac{x(x+m a)^{m-1}}{m !} \mathrm{e}^{-b_{\lambda}(x+m a)}
\end{aligned}
$$

By Theorem 4.1, it follows that

$$
\begin{aligned}
& \mathbb{E}_{x}\left[\mathrm{e}^{-\lambda \tau_{a}^{n-m}} ; X_{\tau_{a}^{n-m}} \in \mathrm{d} y\right] \\
& \quad=\frac{\left(c_{\lambda}\right)^{n-m}(y-x+(n-m) a)^{n-m-1} \mathrm{e}^{-b_{\lambda}(y-x+(n-m) a)} \mathbf{1}_{\{y-x+(n-m) a \geq 0\}}}{(n-m-1) !} \mathrm{d} y .
\end{aligned}
$$

Substituting (4.10) and (4.11) into (4.9) and simplifying, we easily obtain (4.8).

Recall that $\tau_{a}^{1}=\tilde{\tau}_{a}^{1}=\tau_{a}$ and $X_{\tau_{a}}=M_{\tau_{a}}-a$ a.s. Therefore, by letting $\lambda \rightarrow 0+$ and $x=a$ in (4.10), it follows that, for $m=0,1,2, \ldots$,

$$
\begin{aligned}
\mathbb{P}\left\{\tilde{\tau}_{a}^{2}=\tau_{a}^{2+m}\right\} & =\mathbb{P}\left\{\tau_{a}^{m}<T_{a}^{+}<\tau_{a}^{m+1}\right\} \\
& =\frac{(m+1)^{m-1}}{m !}\left(\frac{\gamma a}{\mathrm{e}^{\gamma a}-1}\right)^{m} \mathrm{e}^{-(m+1) \gamma a /\left(\mathrm{e}^{\gamma a}-1\right)},
\end{aligned}
$$

which is the probability mass function of a generalized Poisson RV (see, e.g. Equation (9.1) of Consul and Famoye [7] with $\left.\theta=\lambda=\gamma a /\left(\mathrm{e}^{\gamma a}-1\right)\right)$. For completeness, a RV $Y$ has a generalized Poisson $(\theta, \lambda)$ distribution if its probability mass function $p_{Y}$ is given by

$$
p_{Y}(m)=\frac{\theta(\theta+\lambda m)^{m-1} \mathrm{e}^{-\theta-\lambda m}}{m !}, \quad m=0,1,2, \ldots,
$$

when both $\theta, \lambda>0$.

We will propose a generalization of (4.12) in Theorem 4.3.

Remark 4.1. Equation (4.12) can be interpreted as follows: the number of drawdowns without recovery between two successive drawdowns with recovery follows a generalized Poisson distribution with $\theta=\lambda=\gamma a /\left(\mathrm{e}^{\gamma a}-1\right)$.

In the following result we connect the two drawdown time sequences. It should be noted that the $\mathrm{RV} N_{\tilde{\tau}_{a}^{k}}^{a}-k$ represents the number of drawdowns without recovery over the first $k$ drawdowns with recovery. When $k=2$, (4.13) coincides with (4.12). 
Theorem 4.3. For any $k \in \mathbb{N}, N_{\tilde{\tau}^{k}}^{a}-k$ follows a generalized Poisson distribution with parameters $\theta=(k-1) \gamma a /\left(\mathrm{e}^{\gamma a}-1\right)$ and $\lambda=\gamma a /\left(\mathrm{e}^{\gamma a}-1\right)$, i.e., for $m=0,1,2, \ldots$, it follows that

$$
\begin{aligned}
\mathbb{P}\left\{\tilde{\tau}_{a}^{k}=\tau_{a}^{k+m}\right\} & =\mathbb{P}\left\{N_{\tilde{\tau}_{a}^{k}}^{a}=k+m\right\} \\
& =\frac{k-1}{m+k-1} \frac{\left((m+k-1) \gamma a /\left(\mathrm{e}^{\gamma a}-1\right)\right)^{m}}{m !} \mathrm{e}^{-(m+k-1) \gamma a /\left(\mathrm{e}^{\gamma a}-1\right)} .
\end{aligned}
$$

Proof. It is clear that $\left\{\tilde{\tau}_{a}^{k}=\tau_{a}^{k+m}\right\}$ corresponds to the event that $m$ drawdowns without recovery will occur over the first $k$ drawdowns with recovery, i.e.

$$
\left\{\tilde{\tau}_{a}^{k}=\tau_{a}^{k+m}\right\}=\left\{N_{\tilde{\tau}_{a}^{k}}^{a}=k+m\right\} .
$$

Next we prove that $N_{\tilde{\tau}_{a}^{k}}^{a}-k$ follows a generalized Poisson distribution. By Remark 4.1 and the strong Markov property of $X$, we know that the number of drawdowns without recovery between any two successive drawdowns with recovery are i.i.d. and follow a generalized Poisson distribution with $\theta=\lambda=\gamma a /\left(\mathrm{e}^{\gamma a}-1\right)$. Thus,

$$
N_{\tilde{\tau}_{a}^{k}}^{a}-k=\sum_{i=2}^{k}\left(N_{\tilde{\tau}_{a}^{i}}^{a}-N_{\tilde{\tau}_{a}^{i-1}}^{a}-1\right),
$$

corresponds to a sum of i.i.d. RVs with a generalized Poisson distribution $\theta=\lambda=\gamma a /\left(\mathrm{e}^{\gamma a}-1\right)$. Using Theorem 9.1 of Consul and Famoye [7], we have that $N_{\tilde{\tau}_{a}^{k}}^{a}-k$ follows a generalized Poisson distribution with parameters $\theta=(k-1) \gamma a /\left(\mathrm{e}^{\gamma a}-1\right)$ and $\lambda=\gamma a /\left(\mathrm{e}^{\gamma a}-1\right)$.

Next, we propose the following corollary, which can be viewed as an extension to [20] and [13] from the first drawdown case to the $n$th drawdown without recovery.

Corollary 4.1. For $n \in \mathbb{N}$ and $x>0$, it follows that

$$
\mathbb{E}\left[\mathrm{e}^{-\lambda \tau_{a}^{n}} ; M_{\tau_{a}^{n}}>x\right]=\left(\frac{c_{\lambda}}{b_{\lambda}}\right)^{n} \sum_{m=0}^{n-1} \frac{x(x+m a)^{m-1} b_{\lambda}^{m}}{m !} \mathrm{e}^{-b_{\lambda}(m a+x)} .
$$

Proof. Taking the integral of (4.8) with respect to $y$ in $(-n a, \infty)$, it follows that

$$
\begin{aligned}
& \mathbb{E}\left[\mathrm{e}^{-\lambda \tau_{a}^{n}} ; M_{\tau_{a}^{n}}>x\right] \\
& \quad=\left(c_{\lambda}\right)^{n} \sum_{m=0}^{n-1} \frac{x(x+m a)^{m-1}}{m !(n-m-1) !} \int_{x-(n-m) a}^{\infty} \mathrm{e}^{-b_{\lambda}(y+n a)}(y-x+(n-m) a)^{n-m-1} \mathrm{~d} y \\
& \quad=\left(c_{\lambda}\right)^{n} \sum_{m=0}^{n-1} \frac{x(x+m a)^{m-1}}{m !(n-m-1) !} \int_{0}^{\infty} \mathrm{e}^{-b_{\lambda}(z+x+m a)} z^{n-m-1} \mathrm{~d} z \\
& =\left(c_{\lambda}\right)^{n} \sum_{m=0}^{n-1} \frac{x(x+m a)^{m-1}}{m !(n-m-1) !} \mathrm{e}^{-b_{\lambda}(x+m a)} \int_{0}^{\infty} \mathrm{e}^{-b_{\lambda} z} z^{n-m-1} \mathrm{~d} z \\
& =\left(c_{\lambda}\right)^{n} \sum_{m=0}^{n-1} \frac{x(x+m a)^{m-1}}{m ! b_{\lambda}^{n-m}} \mathrm{e}^{-b_{\lambda}(x+m a)},
\end{aligned}
$$

which completes the proof. 
The marginal distribution of $M_{\tau_{a}^{n}}$ can be easily obtained from Corollary 4.1 by letting $\lambda \rightarrow 0+$ and subsequently making use of (2.6). Indeed,

$$
\mathbb{P}\left\{M_{\tau_{a}^{n}}>x\right\}=\sum_{m=0}^{n-1} \frac{x(x+m a)^{m-1}\left(\gamma /\left(\mathrm{e}^{\gamma a}-1\right)\right)^{m}}{m !} \mathrm{e}^{-\gamma(m a+x) /\left(\mathrm{e}^{\gamma a}-1\right)} .
$$

Rearrangements of (4.14) yield

$$
\mathbb{P}\left\{M_{\tau_{a}^{n}}>x\right\}=\sum_{k=0}^{n-1} D_{k, n} \frac{\left(\gamma x /\left(\mathrm{e}^{\gamma a}-1\right)\right)^{k}}{k !} \mathrm{e}^{-\gamma x /\left(\mathrm{e}^{\gamma a}-1\right)}
$$

where $D_{0, n}=1$, and

$$
\begin{aligned}
D_{k, n} & =\sum_{m=k}^{n-1} \frac{k\left(m \gamma a /\left(\mathrm{e}^{\gamma a}-1\right)\right)^{m-k}}{m(m-k) !} \mathrm{e}^{-m \gamma /\left(\mathrm{e}^{\gamma a}-1\right) a} \\
& =\sum_{m=0}^{n-1-k} \frac{k\left((m+k) \gamma a /\left(\mathrm{e}^{\gamma a}-1\right)\right)^{m}}{(m+k) m !} \mathrm{e}^{-(m+k) \gamma a /\left(\mathrm{e}^{\gamma a}-1\right)}
\end{aligned}
$$

for $k=1,2, \ldots, n-1$. Then,

$$
\mathbb{P}\left\{M_{\tau_{a}^{n}} \in \mathrm{d} y\right\}=\sum_{k=1}^{n} d_{k, n} \frac{\left(\gamma a /\left(\mathrm{e}^{\gamma a}-1\right)\right)^{k} y^{k-1} \mathrm{e}^{-\gamma a y /\left(\mathrm{e}^{\gamma a}-1\right)}}{(k-1) !} \mathrm{d} y,
$$

where $\left\{d_{k, n}\right\}_{k=1}^{n}$ are given by

$$
\begin{aligned}
d_{k, n} \equiv D_{k-1, n}-D_{k, n}= & \sum_{j=k}^{n} \frac{k-1}{j-1} \frac{\left((j-1) \gamma a /\left(\mathrm{e}^{\gamma a}-1\right)\right)^{j-k}}{(j-k) !} \mathrm{e}^{-(j-1) \gamma a /\left(\mathrm{e}^{\gamma a}-1\right)} \\
& \times\left(1-\sum_{m=0}^{n-j-1} \frac{(m+1)^{m-1}}{m !}\left(\frac{\gamma a}{\mathrm{e}^{\gamma a}-1}\right)^{m} \mathrm{e}^{-(m+1) \gamma a /\left(\mathrm{e}^{\gamma a}-1\right)}\right) .
\end{aligned}
$$

In conclusion, $M_{\tau_{a}^{n}}$ follows a mixed Erlang distribution which is an important class of distribution in risk management (see, e.g. Willmot and Lin [22] for an extensive review of mixed Erlang distributions).

Remark 4.2. Note that the distribution of $M_{\tau_{a}^{n}}$ does not come as a surprise. Indeed, we can obtain the structural form of the distribution of $M_{\tau_{a}^{n}}$ by conditioning on $\tilde{N}_{\tau_{a}^{n}}^{a}$, namely, the number of drawdowns with recovery over the first $n$ drawdowns (without recovery). Using the strong Markov property of the process $X$ and (2.7), it follows that $M_{\tau_{a}^{n}} \mid \tilde{N}_{\tau_{a}^{n}}^{a}=m$ is an Erlang RV with mean $m\left(\mathrm{e}^{\gamma a}-1\right) / \gamma$ and variance $m\left(\left(\mathrm{e}^{\gamma a}-1\right) / \gamma\right)^{2}$ for $m=1,2, \ldots, n$. Thus, in (4.15), $D_{k, n}$ can be interpreted as the survival function of $\tilde{N}_{\tau_{a}^{n}}^{a}$, i.e.

$$
D_{k, n}=\mathbb{P}\left\{\tilde{N}_{\tau_{a}^{n}}^{a}>k\right\}=\mathbb{P}\left\{\tilde{\tau}_{a}^{k+1} \leq \tau_{a}^{n}\right\} .
$$

In the next corollary we investigate the actual drawdown $M_{t}-X_{t}$ at $t=\tau_{a}^{n}$. 
Corollary 4.2. For $a \leq x \leq n a$, it follows that

$$
\begin{aligned}
& \mathbb{E}\left[\mathrm{e}^{-\lambda \tau_{a}^{n}} ; M_{\tau_{a}^{n}}-X_{\tau_{a}^{n}} \leq x\right] \\
& =\left(c_{\lambda}\right)^{n} \mathrm{e}^{-b_{\lambda}(n a-x)} \\
& \quad \times \sum_{m=0}^{n-1}\left(\frac{(n a-x)^{m}}{b_{\lambda}^{n-m} m !}-\frac{\mathbf{1}_{\{x \leq(n-m) a\}}((n-m) a-x)^{n-m-1} \int_{0}^{\infty} \mathrm{e}^{-b_{\lambda} y} y(y+m a)^{m-1} \mathrm{~d} y}{m !(n-m-1) !}\right) .
\end{aligned}
$$

Proof. It follows that

$$
\begin{aligned}
\mathbb{E}\left[\mathrm{e}^{-\lambda \tau_{a}^{n}} ; M_{\tau_{a}^{n}}-X_{\tau_{a}^{n}}>x\right] & \\
= & \int_{-x}^{\infty} \mathbb{E}\left[\mathrm{e}^{-\lambda \tau_{a}^{n}} ; M_{\tau_{a}^{n}}-X_{\tau_{a}^{n}}>x, X_{\tau_{a}^{n}} \in \mathrm{d} y\right]+\mathbb{E}\left[\mathrm{e}^{-\lambda \tau_{a}^{n}} ; M_{\tau_{a}^{n}}-X_{\tau_{a}^{n}}>x, X_{\tau_{a}^{n}} \leq-x\right] \\
= & \int_{-x}^{\infty} \mathbb{E}\left[\mathrm{e}^{-\lambda \tau_{a}^{n}} ; M_{\tau_{a}^{n}}>x+y, X_{\tau_{a}^{n}} \in \mathrm{d} y\right]+\mathbb{E}\left[\mathrm{e}^{-\lambda \tau_{a}^{n}} ; X_{\tau_{a}^{n}} \leq-x\right] \\
= & \int_{-x}^{\infty} \mathbb{E}\left[\mathrm{e}^{-\lambda \tau_{a}^{n}} ; M_{\tau_{a}^{n}}>x+y, X_{\tau_{a}^{n}} \in \mathrm{d} y\right] \\
& +\left(\frac{c_{\lambda}}{b_{\lambda}}\right)^{n}\left(1-\mathrm{e}^{-b_{\lambda}(n a-x)} \sum_{m=0}^{n-1} \frac{\left(b_{\lambda}(n a-x)\right)^{m}}{m !}\right),
\end{aligned}
$$

where the last step is due to (4.1). Moreover, by Theorem 4.2, the first term of (4.17) is

$$
\begin{aligned}
\int_{-x}^{\infty} \mathbb{E} & {\left[\mathrm{e}^{-\lambda \tau_{a}^{n}} ; M_{\tau_{a}^{n}}>x+y, X_{\tau_{a}^{n}} \in \mathrm{d} y\right] } \\
= & \left(c_{\lambda}\right)^{n} \sum_{m=0}^{n-1} \frac{((n-m) a-x)^{n-m-1} \mathbf{1}_{\{-x+(n-m) a \geq 0\}}}{m !(n-m-1) !} \\
& \times \int_{-x}^{\infty} \mathrm{e}^{-b_{\lambda}(y+n a)}(x+y)(x+y+m a)^{m-1} \mathrm{~d} y \\
= & \left(c_{\lambda}\right)^{n} \sum_{m=0}^{n-1} \frac{((n-m) a-x)^{n-m-1} \mathbf{1}_{\{x \leq(n-m) a\}}}{m !(n-m-1) !} \int_{0}^{\infty} \mathrm{e}^{-b_{\lambda}(z-x+n a)} z(z+m a)^{m-1} \mathrm{~d} z \\
= & \left(c_{\lambda}\right)^{n} \mathrm{e}^{-b_{\lambda}(n a-x)} \sum_{m=0}^{n-1} \frac{((n-m) a-x)^{n-m-1} \mathbf{1}_{\{x \leq(n-m) a\}}}{m !(n-m-1) !} \int_{0}^{\infty} \mathrm{e}^{-b_{\lambda} z} z(z+m a)^{m-1} \mathrm{~d} z .
\end{aligned}
$$

Substituting this back into (4.17) completes the proof.

To complete the section, we consider a numerical example to compare the distribution of the $n$th drawdown times $\tilde{\tau}_{a}^{n}$ and $\tau_{a}^{n}$ whose Laplace transforms are given in (3.3) and (4.4), respectively. We implement a numerical inverse Laplace transform approach proposed by Abate and Whitt [1]. For ease of notation, we denote the cumulative distribution functions of $\tau_{a}^{n}$ and $\tilde{\tau}_{a}^{n}$ by $F_{n}$ and $\tilde{F}_{n}$, respectively.

The probabilities that at least $n$ drawdowns with or without recovery occur before time 1 for different values of the drift $\mu$ are presented in Table 1 . We observe that $F_{n}(1)>\tilde{F}_{n}(1)$ for $n \geq 2$ due to the relation between $\tau_{a}^{n}$ and $\tilde{\tau}_{a}^{n}$ given in (4.13). In addition, we see that $F_{n}(1)$ increases as $\mu$ decreases. However, we observe the opposite trend for $\tilde{F}_{n}(1)$ when $n \geq 2$. 
TABLE 1: Distribution of the $n$th drawdown times when $a=0.1$ and $\sigma=0.12$.

\begin{tabular}{ccccccc}
\hline & \multicolumn{2}{c}{$\mu=0.1$} & \multicolumn{2}{c}{$\mu=0.0$} & \multicolumn{2}{c}{$\mu=-0.1$} \\
\hline & $F_{n}(1)$ & $\tilde{F}_{n}(1)$ & $F_{n}(1)$ & $\tilde{F}_{n}(1)$ & $F_{n}(1)$ & $\tilde{F}_{n}(1)$ \\
$n=1$ & 0.9779 & 0.9779 & 0.9908 & 0.9908 & 0.9967 & 0.9967 \\
$n=2$ & 0.8759 & 0.4865 & 0.9366 & 0.4406 & 0.9719 & 0.3636 \\
$n=3$ & 0.6651 & 0.1024 & 0.7926 & 0.0885 & 0.8874 & 0.0663 \\
$n=4$ & 0.4060 & 0.0082 & 0.5652 & 0.0070 & 0.7166 & 0.0050 \\
$n=5$ & 0.1942 & 0.0002 & 0.3262 & 0.0002 & 0.4871 & 0.0001 \\
$n=6$ & 0.0721 & 0.0000 & 0.1492 & 0.0000 & 0.2696 & 0.0000 \\
\hline
\end{tabular}

TABLE 2: Distribution of drawdown times when $a=0.1$ and $\sigma=0.12$.

\begin{tabular}{ccccccc}
\hline & \multicolumn{2}{c}{$\mu=0.1$} & \multicolumn{2}{c}{$\mu=0.0$} & \multicolumn{2}{c}{$\mu=-0.1$} \\
\hline & $F_{n}(1)$ & $\tilde{F}_{n}(1)$ & $F_{n}(1)$ & $\tilde{F}_{n}(1)$ & $F_{n}(1)$ & $\tilde{F}_{n}(1)$ \\
$n=1$ & 0.5663 & 0.5663 & 0.7845 & 0.7845 & 0.9257 & 0.9257 \\
$n=2$ & 0.1592 & 0.0339 & 0.3755 & 0.0494 & 0.6509 & 0.0463 \\
$n=3$ & 0.0225 & 0.0002 & 0.0986 & 0.0002 & 0.2891 & 0.0002 \\
$n=4$ & 0.0016 & 0.0000 & 0.0137 & 0.0000 & 0.0730 & 0.0000 \\
$n=5$ & 0.0001 & 0.0000 & 0.0010 & 0.0000 & 0.0099 & 0.0000 \\
$n=6$ & 0.0000 & 0.0000 & 0.0000 & 0.0000 & 0.0007 & 0.0000 \\
\hline
\end{tabular}

This is because the previous running maximum is less likely to be revisited for a smaller $\mu$. Since the drawdown risk is in principle a type of downside risk, it is reasonable to assume that smaller $\mu$ should lead to higher downside risks. In this sense, we suggest that the drawdown times without recovery are a better measure to capture the essence of drawdown risks.

Table 2 is the equivalent of Table 1 but with a lower volatility $\sigma=0.12$. We notice that $F_{n}(1)$ and $\tilde{F}_{n}(1)$ decrease as $\sigma$ decreases. We also have an interesting observation that the trend of $\tilde{F}_{2}(1)$ is not monotone in $\mu$. Again, this is because the occurrence of $\tilde{\tau}_{a}^{n}$ for $n \geq 2$ necessitates a recovery for the previous running maximum. Smaller drift does imply higher drawdown risk; however, the recovery becomes more difficult.

\section{Insurance of frequent relative drawdowns}

In this section we consider insurance policies that protect against the risk of frequent drawdowns. We denote the price of an underlying asset by $S=\left\{S_{t}, t \geq 0\right\}$, with dynamics

$$
\mathrm{d} S_{t}=r S_{t} \mathrm{~d} t+\sigma S_{t} \mathrm{~d} W_{t}^{\mathbb{Q}}, \quad S_{0}=s_{0}>0
$$

where $r>0$ is the risk-free rate, $\sigma>0$, and $\left\{W_{t}^{\mathbb{Q}}, t \geq 0\right\}$ is a standard Brownian motion under a risk-neutral measure $\mathbb{Q}$. It is well known that

$$
S_{t}=s_{0} \mathrm{e}^{X_{t}}
$$

where $X_{t}=\left(r-\frac{1}{2} \sigma^{2}\right) t+\sigma W_{t}^{\mathbb{Q}}$.

In practice, drawdowns are often quoted as a percentage. For fixed $0<\alpha<1$, we denote the time of the first relative drawdown over size $\alpha$ by

$$
\eta_{\alpha}(S)=\inf \left\{t \geq 0: M_{t}^{S}-S_{t} \geq \alpha M_{t}^{S}\right\},
$$

where $M_{t}^{S}=\sup _{0 \leq u \leq t} S_{u}$ represents the running maximum of $S$ by time $t$. By (5.1), it is easy 
to see that the relative drawdown of the geometric Brownian motion $S$ corresponds to the actual drawdown of a drifted Brownian motion $X$, namely,

$$
\eta_{\alpha}(S)=\inf \left\{t \geq 0: M_{t}^{X}-X_{t} \geq-\log (1-\alpha)\right\}=\tau_{\bar{\alpha}}(X),
$$

where $\bar{\alpha}=-\log (1-\alpha)$. Similarly, we denote the relative drawdown times with and without recovery by

$$
\tilde{\eta}_{\alpha}^{n}(S)=\inf \left\{t>\tilde{\eta}_{\alpha}^{n-1}(S): M_{t}^{S}-S_{t} \geq \alpha M_{t}^{S}, M_{t}^{S}>M_{\tilde{\eta}_{\alpha}^{n-1}(S)}^{S}\right\}
$$

and

$$
\eta_{\alpha}^{n}(S)=\inf \left\{t>\eta_{\alpha}^{n-1}(S): M_{\left[\eta_{\alpha}^{n-1}(S), t\right]}^{S}-S_{t} \geq \alpha M_{\left[\eta_{\alpha}^{n-1}(S), t\right]}^{S}\right\}
$$

respectively. Therefore, it follows that

$$
\tilde{\eta}_{\alpha}^{n}(S)=\tilde{\tau}_{\bar{\alpha}}^{n}(X) \quad \text { and } \quad \eta_{\alpha}^{n}(S)=\tau_{\bar{\alpha}}^{n}(X) .
$$

Next, we consider two types of insurance policy that offer a protection against relative drawdowns. For the first policy, we assume that the seller pays the buyer $k$ at time $T$ if a total of $k$ relative drawdowns over size $0<\alpha<1$ occurred prior to time $T$ (for all $k$ ). For the relative drawdown times with and without recovery, by (5.2), the risk-neutral prices are given by

$$
\tilde{V}_{1}(T)=\mathrm{e}^{-r T} \sum_{k=1}^{\infty} k \mathbb{Q}\left\{\tilde{N}_{T}^{\bar{\alpha}}(X)=k\right\}=\mathrm{e}^{-r T} \mathbb{E}^{\mathbb{Q}}\left[\tilde{N}_{T}^{\bar{\alpha}}(X)\right]
$$

and

$$
V_{1}(T)=\mathrm{e}^{-r T} \sum_{k=1}^{\infty} k \mathbb{Q}\left\{N_{T}^{\bar{\alpha}}(X)=k\right\}=\mathrm{e}^{-r T} \mathbb{E}^{\mathbb{Q}}\left[N_{T}^{\bar{\alpha}}(X)\right],
$$

respectively. For the second type of policy, we assume that the seller pays the buyer 1 at the time of each relative drawdown time as long as it occurs before maturity $T$. Hence, their risk-neutral prices are

$$
\tilde{V}_{2}(T)=\sum_{k=1}^{\infty} \mathbb{E}^{\mathbb{Q}}\left[\mathrm{e}^{-r \tilde{\tau}_{\bar{\alpha}}^{k}(X)} ; \tilde{\tau}_{\bar{\alpha}}^{k}(X) \leq T\right]
$$

and

$$
V_{2}(T)=\sum_{k=1}^{\infty} \mathbb{E}^{\mathbb{Q}}\left[\mathrm{e}^{-r \tau \bar{\alpha}(X)} ; \tau_{\bar{\alpha}}^{k}(X) \leq T\right],
$$

respectively.

Corollary 5.1. For $\lambda>0$, it follows that

$$
\begin{aligned}
\int_{0}^{\infty} \mathrm{e}^{-\lambda T} V_{1}(T) \mathrm{d} T & =\frac{1}{\lambda+r} \frac{\bar{c}_{\lambda+r} / \bar{b}_{\lambda+r}}{1-\bar{c}_{\lambda+r} / \bar{b}_{\lambda+r}}, \\
\int_{0}^{\infty} \mathrm{e}^{-\lambda T} \tilde{V}_{1}(T) \mathrm{d} T & =\frac{1}{\lambda+r} \frac{\bar{c}_{\lambda+r} / \bar{b}_{\lambda+r}}{1-\exp \left(-\bar{\beta}_{\lambda+r}^{+} a\right) \bar{c}_{\lambda+r} / \bar{b}_{\lambda+r}}, \\
\int_{0}^{\infty} \mathrm{e}^{-\lambda T} V_{2}(T) \mathrm{d} T & =\frac{1}{\lambda} \frac{\bar{c}_{\lambda+r} / \bar{b}_{\lambda+r}}{1-\bar{c}_{\lambda+r} / \bar{b}_{\lambda+r}}, \\
\int_{0}^{\infty} \mathrm{e}^{-\lambda T} \tilde{V}_{2}(T) \mathrm{d} T & =\frac{1}{\lambda} \frac{\bar{c}_{\lambda+r} / \bar{b}_{\lambda+r}}{1-\exp \left(-\bar{\beta}_{\lambda+r}^{+} a\right) \bar{c}_{\lambda+r} / \bar{b}_{\lambda+r}},
\end{aligned}
$$


where $\bar{b}_{\lambda}=\left(\bar{\beta}_{\lambda}^{+} \mathrm{e}^{-\bar{\beta}_{\lambda}^{-} \bar{\alpha}}-\bar{\beta}_{\lambda}^{-} \mathrm{e}^{-\bar{\beta}_{\lambda}^{+} \bar{\alpha}}\right) /\left(\mathrm{e}^{-\bar{\beta}_{\lambda}^{-} \bar{\alpha}}-\mathrm{e}^{-\bar{\beta}_{\lambda}^{+} \bar{\alpha}}\right), \bar{c}_{\lambda}=\left(\bar{\beta}_{\lambda}^{+}-\bar{\beta}_{\lambda}^{-}\right) /\left(\mathrm{e}^{-\bar{\beta}_{\lambda}^{-} \bar{\alpha}}-\mathrm{e}^{-\bar{\beta}_{\lambda}^{+} \bar{\alpha}}\right)$ and $\bar{\beta}_{\lambda}^{ \pm}=\left(-r+\sigma^{2} / 2 \pm \sqrt{\left(r-\sigma^{2} / 2\right)^{2}+2 \lambda \sigma^{2}}\right) / \sigma^{2}$.

Proof. We provide the proof for $\int_{0}^{\infty} V_{1}(T) \mathrm{e}^{-\lambda T} \mathrm{~d} T$ and $\int_{0}^{\infty} V_{2}(T) \mathrm{e}^{-\lambda T} \mathrm{~d} T$ only. The other two results can be derived in a similar fashion. From the definition of $N_{T}^{\bar{\alpha}}(X)$, we have the following relation

$$
\mathbb{E}^{\mathbb{Q}}\left[N_{T}^{\bar{\alpha}}(X)\right]=\sum_{k=1}^{\infty} \mathbb{Q}\left\{N_{T}^{\bar{\alpha}}(X) \geq k\right\}=\sum_{k=1}^{\infty} \mathbb{Q}\left\{\tau_{\bar{\alpha}}^{k}(X) \leq T\right\} .
$$

By (4.4), it follows that

$$
\begin{aligned}
\int_{0}^{\infty} V_{1}(T) \mathrm{e}^{-\lambda T} \mathrm{~d} T & =\int_{0}^{\infty} \mathrm{e}^{-(\lambda+r) T} \mathbb{E}^{\mathbb{Q}}\left[N_{T}^{\bar{\alpha}}(X)\right] \mathrm{d} T \\
& =\sum_{k=1}^{\infty} \int_{0}^{\infty} \mathrm{e}^{-(\lambda+r) T} \mathbb{Q}\left\{\tau_{\bar{\alpha}}^{k}(X) \leq T\right\} \mathrm{d} T \\
& =\frac{1}{\lambda+r} \sum_{k=1}^{\infty} \mathbb{E}^{\mathbb{Q}}\left[\mathrm{e}^{-(\lambda+r) \tau_{\bar{\alpha}}^{k}(X)}\right] \\
& =\frac{1}{\lambda+r} \sum_{k=1}^{\infty}\left(\frac{\bar{c}_{\lambda+r}}{\bar{b}_{\lambda+r}}\right)^{n} \\
& =\frac{1}{\lambda+r} \frac{\bar{c}_{\lambda+r} / \bar{b}_{\lambda+r}}{1-\bar{c}_{\lambda+r} / \bar{b}_{\lambda+r}} .
\end{aligned}
$$

For $\int_{0}^{\infty} V_{2}(T) \mathrm{e}^{-\lambda T} \mathrm{~d} T$, by Fubini's theorem and (4.4), it follows that

$$
\begin{aligned}
\int_{0}^{\infty} V_{2}(T) \mathrm{e}^{-\lambda T} \mathrm{~d} T & =\sum_{k=1}^{\infty} \int_{0}^{\infty} \mathbb{E}^{\mathbb{Q}}\left[\mathrm{e}^{-r \tau_{\bar{\alpha}}^{k}(X)} ; \tau_{\bar{\alpha}}^{k}(X) \leq T\right] \mathrm{e}^{-\lambda T} \mathrm{~d} T \\
& =\sum_{k=1}^{\infty} \int_{0}^{\infty} \int_{0}^{T} \mathrm{e}^{-r t} \mathbb{Q}\left\{\tau_{\bar{\alpha}}^{k}(X) \in \mathrm{d} t\right\} \mathrm{e}^{-\lambda T} \mathrm{~d} T \\
& =\sum_{k=1}^{\infty} \frac{1}{\lambda} \int_{0}^{\infty} \mathrm{e}^{-(\lambda+r) t} \mathbb{Q}\left\{\tau_{\bar{\alpha}}^{n}(X) \in \mathrm{d} t\right\} \\
& =\sum_{k=1}^{\infty} \frac{1}{\lambda}\left(\frac{\bar{c}_{\lambda+r}}{\bar{b}_{\lambda+r}}\right)^{n} \\
& =\frac{1}{\lambda} \frac{\bar{c}_{\lambda+r} / \bar{b}_{\lambda+r}}{1-\bar{c}_{\lambda+r} / \bar{b}_{\lambda+r}}
\end{aligned}
$$

This completes the proof.

Remark 5.1. It is worth pointing out that, through expansion of the randomized prices in Corollary 5.1 in terms of exponentials, it is possible to obtain semistatic hedging portfolios as in [5]. Moreover, capped insurance contracts against frequency of drawdowns can also be formulated and priced using Theorems 3.1 and 4.1, and Corollary 4.1. 
TABLE 3: Insurance contract prices when $\alpha=15 \%$ and $r=5 \%$.

\begin{tabular}{llllll}
\hline & & $V_{1}(T)$ & $\tilde{V}_{1}(T)$ & $V_{2}(T)$ & $\tilde{V}_{2}(T)$ \\
\hline$T=1$ & $\sigma=0.1$ & 0.1102 & 0.1091 & 0.1120 & 0.1108 \\
$T=2$ & $\sigma=0.1$ & 0.3011 & 0.2769 & 0.3131 & 0.2885 \\
$T=3$ & $\sigma=0.1$ & 0.4743 & 0.4031 & 0.5058 & 0.4318 \\
\hline$T=1$ & $\sigma=0.2$ & 1.1777 & 0.7873 & 1.2043 & 0.8081 \\
$T=2$ & $\sigma=0.2$ & 2.3815 & 1.1842 & 2.4977 & 1.2550 \\
$T=3$ & $\sigma=0.2$ & 3.4651 & 1.4519 & 3.7279 & 1.5890 \\
\hline
\end{tabular}

To conclude, we consider a pricing example for the four types of insurance contract proposed earlier. The same numerical Laplace transform approach as in the last section is applied; see Table 3.

As we expect, type 2 contracts have higher prices than type 1 contracts because of earlier payments (at the moment of each drawdown time instead of the maturity $T$ ). From Table 3 we also show that $\tilde{V}_{1}(T)$ and $\tilde{V}_{2}(T)$ are respectively lower than $V_{1}(T)$ and $V_{2}(T)$ due to $\tau_{a}^{n} \leq \tilde{\tau}_{a}^{n}$. All the prices increase as $T$ increases or $\sigma$ increases. Moreover, we can expect that the prices will decrease as $\alpha$ or $r$ increases. The latter is due to a higher discount rate which is the risk-free rate under the risk-neutral measure $\mathbb{Q}$.

\section{Acknowledgements}

The authors would like to thank Professor Gord Willmot and an anonymous referee for their helpful remarks and suggestions. David Landriault and Bin Li gratefully acknowledge the support from a grant from the Natural Sciences and Engineering Research Council of Canada and a start-up grant from the University of Waterloo, respectively.

\section{References}

[1] AвAte, J. AND Whitt, W. (2006). A unified framework for numerically inverting Laplace transforms. INFORMS J. Comput. 18, 408-421.

[2] Andersen, E. S. (1957). On the collective theory of risk in the case of contagion between the claims. In Transactions of the XVth International Congress of Actuaries, Vol. II, New York, pp. 104-125.

[3] Borodin, A. and Salminen, P. (2002). Handbook of Brownian Motion-Facts and Formulae, 2nd edn. Birkhäuser, Basel.

[4] Burghardt, G., Duncan, R. and Liu, L. (2003). Deciphering drawdown. Risk magazine 5, S16-S20.

[5] Carr, P., Zhang, H. and HadjILIAdis, O. (2011). Maximum drawdown insurance. Internat. J. Theoret. Appl. Finance 14, 1195-1230.

[6] Chekhlov, A., Uryasev, S. and Zabarankin, M. (2005). Drawdown measure in portfolio optimization. Internat. J. Theoret. Appl. Finance 8, 13-58.

[7] Consul, P. C. and Famoye, F. (2006). Lagrangian Probability Distributions. Birkhäuser, Boston, MA.

[8] Doundy, R., Shiryaev, A. N. AND Yor, M. (2000). On probability characteristics of 'downfalls' in a standard Brownian motion. Theory Prob. Appl. 44, 29-38.

[9] Grossman, S. J. And Zhou, Z. (1993). Optimal investment strategies for controlling drawdowns. Math. Finance 3, 241-276.

[10] Hadjiliadis, O. ANd VeČĚ̌, J. (2006). Drawdowns preceding rallies in a Brownian motion model. Quant. Finance 6, 403-409.

[11] Hamelink, F. And Hoesli, M. (2004). Maximum drawdown and the allocation to real estate. J. Property Res. 21, 5-29.

[12] Jarrow, R., Kchia, Y. And Protter, P. (2011). How to detect an asset bubble. SiAM J. Financial Math. 2, 839-865.

[13] LehoczKy, J. P. (1977). Formulas for stopped diffusion processes with stopping times based on the maximum. Ann. Prob. 5, 601-607. 
[14] Magdon-Ismail, M., Atiya, A. F., Pratap, A. and Abu-Mostafa, Y. S. (2004). On the maximum drawdown of a Brownian motion. J. Appl. Prob. 41, 147-161.

[15] Mijatović, A. AND Pistorius, M. R. (2012). On the drawdown of completely asymmetric Lévy processes. Stoch. Process. Appl. 122, 3812-3836.

[16] Pfeffer, D. (2001). Sequential barrier options. Algo Res. Quart. 4, 65-74.

[17] Pospisil, L. AND VEČĚ̌, J. (2010). Portfolio sensitivity to changes in the maximum and the maximum drawdown. Quant. Finance 10, 617-627.

[18] Pospisil, L., VeČĚ̌, J. AND HadjILIAdis, O. (2009). Formulas for stopped diffusion processes with stopping times based on drawdowns and drawups. Stoch. Process. Appl. 119, 2563-2578.

[19] Rolski, T., Schmidli, H., Schmidt, V. And Teugels, J. (1999). Stochastic Processes for Insurance and Finance. John Wiley, Chichester.

[20] Taylor, H. M. (1975). A stopped Brownian motion formula. Ann. Prob. 3, 234-246.

[21] VeČĚ̌, J. (2006). Maximum drawdown and directional trading. Risk 19, 88-92.

[22] Willmot, G. E. And Lin, X. S. (2011). Risk modelling with the mixed Erlang distribution. Appl. Stoch. Models Business Industry 27, 8-22.

[23] Zhang, H. (2015). Occupation times, drawdowns, and drawups for one-dimensional regular diffusions. Adv. Appl. Prob. 1, 209-229.

[24] Zhang, H. And Hadjiliadis, O. (2010). Drawdowns and rallies in a finite time-horizon. Drawdowns and rallies. Methodol. Comput. Appl. Prob. 12, 293-308.

[25] Zhang, H. and Hadjiliadis, O. (2012). Drawdowns and the speed of market crash. Methodol. Comput. Appl. Prob. 14, 739-752.

[26] Zhang, H., Leung, T. and Hadjiliadis, O. (2013). Stochastic modeling and fair valuation of drawdown insurance. Insurance Math. Econom. 53, 840-850. 